\title{
Acute Lower Limb Ischemia Revealing Myxoma of the Left Atrium: A Case Report and Review of the Literature
}

\author{
Elfatemi. $\mathrm{B}^{1^{*}}$, Tamdy. $\mathrm{A}^{2}$, Belhaj. $\mathrm{S}^{3}$
}

${ }^{1}$ Department of Vascular Surgery, The Regional Hospital Mohamed V in Tangier, 1 Avenue Moulay Rachid, Tanger 90100, Morocco

${ }^{2}$ Department of Cardiology, The Duc De Tovar Hospital in Tangier, Morocco

${ }^{3}$ Agdal Clinic Rabat, Morocco

DOI: $10.36347 /$ sasjs.2020.v06i03.012

| Received: 09.03.2020 | Accepted: 16.03.2020 | Published: 19.03.2020

*Corresponding author: Elfatemi Bouthainah

\section{Abstract}

The observation of a 52-year-old hypertensive patient is reported. This is a patient undergoing treatment admitted to the emergency department for severe acute ischemia of the left lower limb with coldness of the entire leg, cyanosis and sensory-motor paralysis. An angioscanner exam was carried out to objective a total thrombosis of the left primary iliac artery without revision. The patient was admitted to the operating room, having had an embolectomy by a left Scarpa approach, gelatinous thrombus was recovered from the iliac and fibrino-cruoric thrombus from the superficial and deep femoral artery, due to the severe ischemia it was considered to perform discharge fasciotomies to avoid the risk of postoperative re-vascularization syndrome. In view of the yellowish and gelatinous nature of the thrombus, histological examination was performed confirming the presence of myxoid cells. The patient had very good functional progress having recovered the motor skills of the toes, and at day 5, he had his myxoma surgically removed. The postoperative follow-up was without any particularity, a regular scanner exam was recommended in order to detect any possible tumor recurrence. Observations reported in the literature have described cases of aortic thrombosis with bilateral ischemia and their evolution was marked by death due to re-vascularization syndrome [3-6]. This observation is reported in order to remind surgeons of the embolic nature of atrial myxoma due to its friability and to encourage them to analyze the nature of the thrombus for early diagnosis and timely management.

Keywords: Acute ischemia - embolectomy - gelatinous thrombus - atrial fibromyxoma - surgical exeresis.

Copyright @ 2020: This is an open-access article distributed under the terms of the Creative Commons Attribution license which permits unrestricted use, distribution, and reproduction in any medium for non-commercial use (NonCommercial, or CC-BY-NC) provided the original author and source are credited.

\section{INTRODUCTION}

Primary tumors of the heart represents less than $0.1 \%$ of all heart disease [1], with myxomas being the most common form and located in $75 \%$ of cases in the left atrium. They are benign tumors that are often asymptomatic but gelatinous and friable in nature. Their major risk is arterial embolization, especially in the visceral arteries, arteries destined for the brain and those of the limbs. The observation of a patient with severe ischemia of the left lower limb revealing an atrial myxoma is reported.

\section{OBSERVATION}

The case of a 52-year-old hypertensive patient undergoing treatment to have presented to the emergency department with severe pain, sensory-motor paralysis and pulse suppression in the left lower extremity suggestive of ischemia is reported. The angioscanner exam concluded that there was total thrombosis of the left primary iliac artery and the right leg axes. A Fogarty catheter thrombectomy by Scarpa approach was performed on the left superficial and deep femoral arteries bringing back fresh thrombus as well as yellowish and gelatinous material from the left iliac axis and discharge aponevrotomies due to severe ischemia. Post-operatively the patient did not present a revascularization syndrome and received rehydration with saline and bicarbonate serum, the histological analysis completed by the immune-histochemical study concluded to a myxoma of cardiac origin with no malignancy signs. Trans-thoracic cardiac echograph scan confirmed the presence of a tissue mass in the left atrium suspected of myxoma (Figure-1). The standard biological, immunological and thrombophilia test were normal. The patient was transferred for open-heart excision surgery five days later (Figure-2).

The progression was good. Regular transthoracic cardiac echograph scan follow-up was necessary to detect any tumor recurrence. 


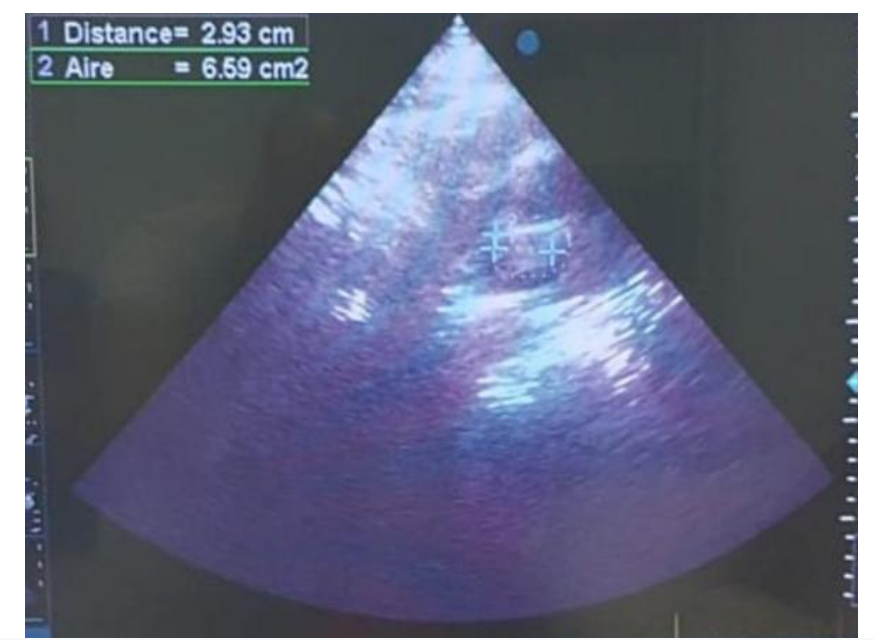

Fig-1: Transthoracic ultrasound showing a suspicious image of a Myxoma
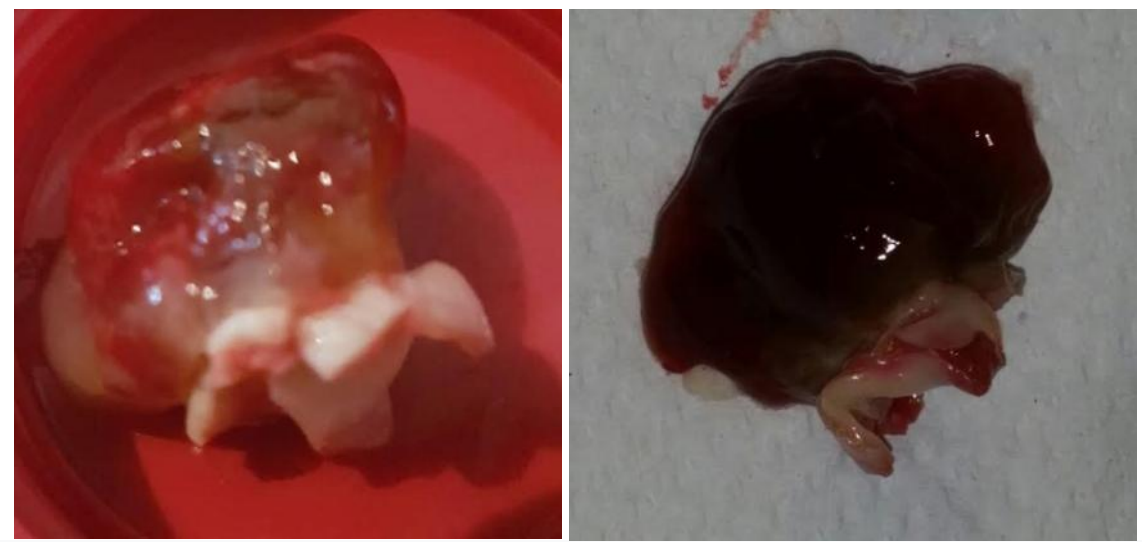

Fig-2: Macroscopic appearance of the Myxoma after surgical resection

\section{DISCUSSION}

Acute lower extremity ischemia is a surgical emergency. The most frequent cause is embolism of cardiac origin (valvuloplasties, atrial fibrillation arrhythmia).

Myxoma of the left atrium is a rare etiology. Its clinical manifestations are polymorphic and several clinical signs may be detected: a pseudo-mitral picture, syncopes and lipothymtias, a febrile picture simulating endocarditis, or systemic emboli [1]. Their symptomatology is variable, depending on the location, shape and size of these tumors [2]. They can manifest themselves by palpitations, or signs of heart failure, as well as by embolic complications, as is the case in the patient reported below.

Electrically, there may be arrhythmia, atrial fibrillation, tachycardia or signs of atrial hypertrophy. It is a benign tumor but only histologically, as it is responsible for life-threatening complications. The embolic phenomenon due to cardiac myxoma is the most frequent complication and can be fatal. It is the result of fragmentation or complete migration of the tumor due to its friable nature and occurs in $30-40 \%$ of cases [3]. Most of these tumor emboli involve the cerebral and visceral arteries.
Clinically, acute lower limb ischemia manifests itself by coldness, intense pain in the lower limb with or without sensory-motor paralysis depending on the time, objective vascular examination and pulse suppression. The angioscanner exam is the reference examination [4] and allows to set a positive diagnosis as well as the search for other locations, especially in the visceral arteries and the contralateral limb. The treatment of acute ischemia is surgical by embolectomy of the scarpa, as the gelatinous nature of the scarpa requires a histological examination of the embolus in order to allow early diagnosis and appropriate management. Post-operatively Trans-thoracic echocardiography (TTE) is routinely carried out and allows the diagnosis of myxoma, but the absence of a tissue image during TTE does not eliminate the diagnosis of occlusion in myxoma by complete migration of the tumor [3-6]. The prognosis is serious, it is essentially linked to the delay in treatment with the consequences of re-vascularization (hyper-kalemia, acute renal failure) $[3,5,7]$, the prognosis can only be improved by an early and multidisciplinary management with adapted operative measures: discharge fasciotomies as in the case of this patient. The treatment of myxoma of the left atrium is emergency total open-heart surgical resection in order to avoid recurrence of emboli. Myxoma surgery is generally 
safe. Post-operative monitoring with regular transthoracic echocardiography scans allows monitoring and screening for any tumor recurrence.

\section{CONCLUSION}

Acute ischemia of the lower limb is a fairly common consequence of atrial myxoma. An early diagnosis and good management, especially histological examination of the embolic material, may prevent any complication and reduce the mortality rate associated with theses complications.

\section{REFERENCES}

1. Frieh JP, Ninet J, El Kirat M, Sassolas F, Gressier M. Les tumeurs primitives du cœur: aspects diagnostiques, anatomiques et thérapeutiques. Archives des maladies du coeur et des vaisseaux. 1986;79(8):1188-94.

2. Wang JG, Li YJ, Liu H, Li NN, Zhao J, Xing XM. Clinicopathologic analysis of cardiac myxomas: seven years' experience with 61 patients. Journal of thoracic disease. 2012 Jun 1;4(3):272.
3. Ohgo T, Yamamoto K, Furuno T. Complete detachment of cardiac myxoma causing aortic saddle embolization and cerebral infarction. International journal of cardiology. $2008 \mathrm{Jul}$ 4;127(2):e48-9.

4. Surowiec SM, Isiklar H, Sreeram S, Weiss VJ, Lumsden AB. Acute occlusion of the abdominal aorta. The American journal of surgery. 1998 Aug 1;176(2):193-7.

5. Neff CM, McCowan CL. Complete aortic occlusion caused by cardiac myxoma emboli. The American journal of emergency medicine. 2008 Jan 1;26(1):110-e1.

6. Guthrie J, Fairgrieve J. Aortic embolism due to myxoid tumour associated with myocardial calcification. British Heart Journal, 1963;25:13740.

7. Ali T, Castro J, Young CR, Burnand KG. Complications of reperfusion in acute aortic artery occlusion following saddle embolization originating from an atrial myxoma. Vascular, 2004;12:202-5. 